\title{
ON PAIRWISE $b$-LOCALLY OPEN AND PAIRWISE $b$-LOCALLY CLOSED FUNCTIONS IN BITOPOLOGICAL SPACES
}

\author{
BINOD CHANDRA TRIPATHY AND DIGANTA JYOTI SARMA
}

\begin{abstract}
The aim of this paper is to introduce and study pairwise $b$-locally open and pairwise $b$-locally closed functions in bitopological spaces and some characterization and several properties concerning these concepts are investigated.
\end{abstract}

\section{Introduction and preliminaries}

The study of bitopological spaces was first initiated by Kelly [4] and thereafter a large numbers of papers have been done to generalize the topological concepts to bitopological setting. Andrijevic [1] defined the notion of $b$-open sets in topological spaces. A set $A$ is said to be $b$-open if $A \subset \operatorname{cl}(\operatorname{int}(A)) \cup \operatorname{int}(\operatorname{cl}(A))$. The complement of $b$-open set is called $b$-closed.

The notion of locally closedness was first introduced by Kurotowski and Sierpienski [5]. There after Nasef [6] introduced and studied $b$-locally closed sets in topological spaces. Rajesh [8] generalized the concepts of $b$-locally closed sets in bitopological setting as follows.

Definition 1.1. A subset $A$ of a bitopological space $\left(X, \tau_{1}, \tau_{2}\right)$ is called $\left(\tau_{1}, \tau_{2}\right)$-b-locally closed (in short $\left(\tau_{1}, \tau_{2}\right)$ - $\left.b L C\right)$ if $A=P \cap Q$ where $P$ is $\tau_{1}$-b-open and $Q$ is $\tau_{2}$-b-closed in $\left(X, \tau_{1}, \tau_{2}\right)$.

Recently Tripathy and Sarma [9] have introduced the notion of $b$-locally open sets in bitopological spaces as follows.

Definition 1.2. A subset $A$ of a bitopological space $\left(X, \tau_{1}, \tau_{2}\right)$ is called $\left(\tau_{1}, \tau_{2}\right)$-b-locally open (in short $\left(\tau_{1}, \tau_{2}\right)$ - $\left.b L O\right)$ if $A=P \cup Q$, where $P$ is $\tau_{1}$-b-closed and $Q$ is $\tau_{2}$-b-open in $\left(X, \tau_{1}, \tau_{2}\right)$.

Tripathy and Sarma [10] have introduced the following definitions.

Definition 1.3. The $\left(\tau_{1}, \tau_{2}\right)$ - $b$-locally interior of a set $A$ is denoted by $\left(\tau_{1}, \tau_{2}\right)-b L \operatorname{int}(A)$ and defined as $\left(\tau_{1}, \tau_{2}\right)$ - $b L$ int $(A)=\cup\left\{B: B\right.$ is $\left(\tau_{1}, \tau_{2}\right)$ - $b$-locally open set and $\left.B \subset A\right\}$.

Corresponding author: Binod Chandra Tripathy.

2010 Mathematics Subject Classification. 54A05, 54A10, 54D20, 54C05, 54C08, 54C10.

Key words and phrases. Bitopological spaces, $b$-open sets, $b$-closed sets, pairwise $b$-locally open, pairwise $b$-locally closed. 
Definition 1.4. The $\left(\tau_{1}, \tau_{2}\right)$ - $b$-locally closure of a set $A$ is denoted by $\left(\tau_{1}, \tau_{2}\right)-b L \operatorname{cl}(A)$ and defined as $\left(\tau_{1}, \tau_{2}\right)-b L \operatorname{cl}(A)=\cap\left\{B: B\right.$ is $\left(\tau_{1}, \tau_{2}\right)$ - $b$-locally closed set and $\left.A \subset B\right\}$.

In view of the above definitions we have the following results.

(i) $\left(\tau_{1}, \tau_{2}\right)-b L \operatorname{int}(A) \subset A$ and $\left(\tau_{1}, \tau_{2}\right)-b L \operatorname{cl}(A) \subset A$.

(ii) $A \subset B \Longrightarrow\left(\tau_{1}, \tau_{2}\right)-b L \operatorname{int}(A) \subset\left(\tau_{1}, \tau_{2}\right)-b L \operatorname{int}(B)$ and $\left(\tau_{1}, \tau_{2}\right)-b L \operatorname{cl}(A) \subset\left(\tau_{1}, \tau_{2}\right)-b L \operatorname{cl}(B)$.

Remark 1.1 (Tripathy and Sarma [10] Remark 2.1). A set $A$ is $\left(\tau_{1}, \tau_{2}\right)$-b-locally open if $\left(\tau_{1}, \tau_{2}\right)$ $b L \operatorname{int}(A)=A$ and $\left(\tau_{1}, \tau_{2}\right)$-b-locally closed if $\left(\tau_{1}, \tau_{2}\right)-b L \operatorname{cl}(A)=A$.

The following definition is due to Pervin [7].

Definition 1.5. A function $f:\left(X, \tau_{1}, \tau_{2}\right) \longrightarrow\left(Y, \sigma_{1}, \sigma_{2}\right)$ is said to be pairwise continuous if the induced functions $f:\left(X, \tau_{1}\right) \rightarrow\left(Y, \sigma_{1}\right)$ and $f:\left(X, \tau_{2}\right) \rightarrow\left(Y, \sigma_{2}\right)$ are both continuous.

In this paper, we introduce the notion of pairwise $b$-locally open function and pairwise $b$-locally closed function in bitopological spaces and investigate the properties of these functions.

Throughout $\left(X, \tau_{1}, \tau_{2}\right)$ will denote a bitopological space on which no separation axioms are assumed. For a subset $A$ of a bitopological space $\left(X, \tau_{1}, \tau_{2}\right), \tau_{i}$-cl $(A)\left(\operatorname{resp} . \tau_{i}\right.$-int $\left.(A)\right)$ denotes the closure (resp. interior) of $A$ with respect to $\tau_{i}$ for $i=1,2$.

The collection of all $\left(\tau_{1}, \tau_{2}\right)-b L O$ sets [resp. $\left(\tau_{1}, \tau_{2}\right)-b L C$ sets] of $\left(X, \tau_{1}, \tau_{2}\right)$ will be denoted by $\left(\tau_{1}, \tau_{2}\right)-b L O(X)\left[\operatorname{resp} .\left(\tau_{1}, \tau_{2}\right)-b L C(X)\right]$.

\section{Pairwise $b$-locally open mapping}

Definition 2.1. Let $\left(X, \tau_{1}, \tau_{2}\right)$ and $\left(Y, \sigma_{1}, \sigma_{2}\right)$ be two bitopological spaces. A function $f:(X$, $\left.\tau_{1}, \tau_{2}\right) \longrightarrow\left(Y, \sigma_{1}, \sigma_{2}\right)$ is called pairwise $b$-locally open (in short, pairwise $\left.b L O\right)$ mapping if the image of each $\left(\tau_{1}, \tau_{2}\right)$-b-locally open set in $X$ is $\sigma_{i}$-open set in $Y$, where $i=1,2$.

Example 2.1. Let $X=Y=\{a, b, c\}, \tau_{1}=\{\varnothing,\{b\}, X\}, \tau_{2}=\{\varnothing,\{a\}, X\}, \sigma_{1}=\{\varnothing,\{b\},\{c\},\{b, c\}, Y\}$, $\sigma_{2}=\{\varnothing,\{c\},\{b, c\}, Y\}$. Consider the function $f:\left(X, \tau_{1}, \tau_{2}\right) \longrightarrow\left(Y, \sigma_{1}, \sigma_{2}\right)$ defined by $f(a)=$ $b, f(b)=c, f(c)=c$. Here $\left(\tau_{1}, \tau_{2}\right)-b L O$ sets are $\{\varnothing,\{a\},\{c\},\{a, b\},\{a, c\}, X\}$ and image of each $\left(\tau_{1}, \tau_{2}\right)-b L O$ sets are $\sigma_{1}$-open. Also $\left(\tau_{2}, \tau_{1}\right)$-bLO sets are $\{\varnothing,\{b\},\{c\},\{a, b\},\{b, c\}, X\}$ and image of each $\left(\tau_{2}, \tau_{1}\right)$-bLO sets are $\sigma_{2}$-open. Hence $f$ is pairwise $b$-locally open mapping.

Theorem 2.1. Let $f:\left(X, \tau_{1}, \tau_{2}\right) \longrightarrow\left(Y, \sigma_{1}, \sigma_{2}\right)$ be a mapping between two bitopological spaces. Then the following are equivalent:

(a) $f$ is pairwise b-locallyopen mapping. 
(b) $f\left(\left(\tau_{1}, \tau_{2}\right)-b L \operatorname{int}(A)\right) \subset \sigma_{i}$-int $(f(A))$, for every subset $A$ of $X$, where $i=1,2$.

Proof. (a) $\Longrightarrow$ (b) Since $\left(\tau_{1}, \tau_{2}\right)-b L$ int $(A)$ is a $\left(\tau_{1}, \tau_{2}\right)$ - $b L O$ set in $X$ for any subset $A$ of $X$ and $\left(\tau_{1}, \tau_{2}\right)-b L \operatorname{int}(A) \subset A$. Then we have $f\left(\left(\tau_{1}, \tau_{2}\right)-b L \operatorname{int}(A)\right) \subset f(A)$.

Since $f$ is pairwise $b L O$-mapping, therefore $f\left(\left(\tau_{1}, \tau_{2}\right)\right.$ - $\left.b L \operatorname{int}(A)\right)$ is $\sigma_{i}$-open.

Hence $f\left(\left(\tau_{1}, \tau_{2}\right)-b L \operatorname{int}(A)\right) \subset \sigma_{i}-\operatorname{int}(f(A))$.

(b) $\Longrightarrow$ (a) Let $A$ be a $\left(\tau_{1}, \tau_{2}\right)-b L O$ set in $X$.

We have $\sigma_{i}$-int $(f(A)) \subset f(A)$.

By hypothesis $f\left(\left(\tau_{1}, \tau_{2}\right)-b L \operatorname{int}(A)\right) \subset \sigma_{i}$-int $(f(A)) \Longrightarrow f(A) \subset \sigma_{i}$-int $(f(A))$

Therefore from (1) and (2), we get $f(A)$ is $\sigma_{i}$-open in $Y$. Hence $f$ is pairwise $b$-locally open map.

Definition 2.2. A subset $S$ is called an $\left(\tau_{1}, \tau_{2}\right)$-b-locally open neighbourhood of a point $x$ of $\left(X, \tau_{1}, \tau_{2}\right)$ if there exists a $\left(\tau_{1}, \tau_{2}\right)$-b-locally open set $V$ such that $x \in V \subset S$.

Theorem 2.2. Let $f:\left(X, \tau_{1}, \tau_{2}\right) \rightarrow\left(Y, \sigma_{1}, \sigma_{2}\right)$ be a function. Then the following are equivalent.

(a) $f$ is pairwise b-locally open mapping.

(b) For each $x \in X$ and each $\left(\tau_{1}, \tau_{2}\right)$-b-locally open neighbourhood $A$ of $x$ in $X$, there exists $a$ $\sigma_{i}$-neighbourhood $C$ of $f(x)$ such that $C \subset f(A)$, where $i=1,2$.

Proof. (a) $\Longrightarrow$ (b) Let $A$ be a $\left(\tau_{1}, \tau_{2}\right)$-b-locally open neighbourhood of $x$ and $x \in X$. Therefore there exists a $\left(\tau_{1}, \tau_{2}\right)$-b-locally open set $B$ in $X$ such that $x \in B \subset A$. Since $f$ is pairwise $b$ locally open map, so by Theorem 2.1., we have

$$
f\left(\left(\tau_{1}, \tau_{2}\right)-b L \operatorname{int}(B)\right) \subset \sigma_{i}-\operatorname{int}(f(B)) \Longrightarrow f(B) \subset \sigma_{i}-\operatorname{int}(f(B)) .
$$

Hence $f(B)$ is $\sigma_{i}$-open set such that $f(x) \in f(B) \subset f(A)$.

Putting $f(B)=C$, we get $C$ is a $\sigma_{i}$-open set such that $C \subset f(A)$.

(b) $\Longrightarrow$ (a) Let $A$ be a $\left(\tau_{1}, \tau_{2}\right)$-b-locally open set in $X$ and $x \in X$. By hypothesis, for each $f(x) \in$ $f(A)$, there exists a $\sigma_{i}$-neighbourhood $B_{f(x)}$ of $f(x)$ such that $B_{f(x)} \subset f(A)$.

Since $B_{f(x)}$ is $\sigma_{i}$-neighbourhood of $f(x)$, there exists a $\sigma_{i}$-open set $C_{f(x)}$ such that $f(x) \in$ $C_{f(x)} \subset B_{f(x)}$.

Now $f(A)=\cup\left\{C_{f(x)}: f(x) \in f(A)\right\}$. It is clear that $f(A)$ is $\sigma_{i}$-open.

Hence $f$ is pairwise $b$-locally open mapping. 
Theorem 2.3. Let, $f:\left(X, \tau_{1}, \tau_{2}\right) \rightarrow\left(Y, \sigma_{1}, \sigma_{2}\right)$ and $g:\left(Y, \sigma_{1}, \sigma_{2}\right) \rightarrow\left(Z, \theta_{1}, \theta_{2}\right)$ be two mappings. If $g_{0} f: X \rightarrow Z$ is pairwise $b$-locally open map and $g$ is pairwise continuous injection, then $f$ is pairwise b-locally open map.

Proof. Let $A$ be an $\left(\tau_{1}, \tau_{2}\right)$-b-locally open set in $X$. Since $g_{0} f$ is pairwise $b$-locally open map, therefore $\left(g_{0} f\right)(A)$ is $\theta_{i}$-open.

Further $g$ is pairwise continuous and injective, therefore $g^{-1}\left(g(f(A))=f(A)\right.$ is $\sigma_{i}$-open. Hence $f$ is pairwise $b$-locally open mapping.

Theorem 2.4. A function $f:\left(X, \tau_{1}, \tau_{2}\right) \rightarrow\left(Y, \sigma_{1}, \sigma_{2}\right)$ is pairwise b-locally open if and only iffor any subset $A$ of $Y$ and for any $\left(\tau_{1}, \tau_{2}\right)$-b-locally closed set $B$ in $X$ such that $f^{-1}(A) \subset B$, then there exists a $\sigma_{i}$-closed set $C$ containing A such that $f^{-1}(C) \subset B$, where $i=1,2$.

Proof. Let $G$ be a $\left(\tau_{1}, \tau_{2}\right)$ - $b$-locally open set in $\left(X, \tau_{1}, \tau_{2}\right)$.

$$
\text { Put } \quad A=Y-f(G) \Longrightarrow f^{-1}(A)=f^{-1}(Y)-G=X-G \text {. }
$$

Then $X-G$ is a $\left(\tau_{1}, \tau_{2}\right)$ - $b$-locally closed set in $X$ such that $f^{-1}(A) \subset X-G$. By hypothesis, there exists a $\sigma_{i}$-closed set $C$ containing $A$ such that

$$
\begin{aligned}
f^{-1}(C) \subset X-G & \Rightarrow C \subset f(X)-f(G)=Y-f(G) \\
& \Longrightarrow f(G) \subset Y-C .
\end{aligned}
$$

Since $A \subset C$, we have $Y-C \subset Y-A=f(G)$, by (3)

$$
\Longrightarrow Y-C \subset f(G) .
$$

Therefore from (4) and (5), we get $f(G)=Y-C$ and so $f(G)$ is $\sigma_{i}$-open since $C$ is $\sigma_{i^{-}}$closed.

Hence $f$ is pairwise $b$-locally open mapping.

Conversely, let $A$ be any subset of $Y$ and $B$ be a $\left(\tau_{1}, \tau_{2}\right)$ - $b$-locally closed set of $X$ such that $f^{-1}(A) \subset B$.

Suppose that $f$ is pairwise $b$-locally open map. Let $C=Y-f(X-B)$.

Since $B$ is $\left(\tau_{1}, \tau_{2}\right)$ - $b$-locally closed set in $X$, so $X-B$ is $\left(\tau_{1}, \tau_{2}\right)$-b-locally open set in $X$.

Since $f$ is pairwise $b$-locally open, therefore $f(X-B)$ is $\sigma_{i}$-open.

$\Longrightarrow Y-f(X-B)$ is $\sigma_{i}$-closed

$\Longrightarrow C$ is $\sigma_{i}$-closed. 
Now, $C=Y-f(X-B)$

$$
\begin{aligned}
& \Longrightarrow f^{-1}(C)=X-(X-B) \subset B \\
& \Longrightarrow f^{-1}(C) \subset B
\end{aligned}
$$

Since $f^{-1}(A) \subset B$

$$
\begin{aligned}
& \Longrightarrow X-f^{-1}(A) \supset X-B \\
& . \Longrightarrow Y-A \supset f(X-B) \\
& \Longrightarrow A \subset Y-f(X-B)=C \\
& \Longrightarrow A \subset C
\end{aligned}
$$

Thus there exists a $\sigma_{i}$-closed set $C$ such that $f^{-1}(C) \subset B$.

Theorem 2.5. Let $f:\left(X, \tau_{1}, \tau_{2}\right) \rightarrow\left(Y, \sigma_{1}, \sigma_{2}\right)$ be a mapping. Then the following are equivalent.

(a) $f$ is pairwise b-locally open mapping.

(b) $f^{-1}\left(\sigma_{i}-\operatorname{cl}(A)\right) \subset\left(\tau_{1}, \tau_{2}\right)-b L \operatorname{cl}\left(f^{-1}(A)\right)$ for every subset $A$ of $Y$, where $i=1,2$.

Proof. (a) $\Longrightarrow$ (b) Let $A$ be a subset of $Y$. Therefore we have $f^{-1}(A) \subset\left(\tau_{1}, \tau_{2}\right)-b L \operatorname{cl}\left(f^{-1}(A)\right)$ and $\left(\tau_{1}, \tau_{2}\right)-b L \operatorname{cl}\left(f^{-1}(A)\right)$ is $\left(\tau_{1}, \tau_{2}\right)$-b-locally closed set in $X$. Since $f$ is pairwise $b$-locally open mapping, so by Theorem 2.4, there exists a $\sigma_{i}$-closed set $B$ such that $A \subset B$ and $f^{-1}(B) \subset$ $\left(\tau_{1}, \tau_{2}\right)-b L \operatorname{cl}\left(f^{-1}(A)\right)$.

Also $A \subset B$

$$
\Longrightarrow f^{-1}(A) \subset f^{-1}(B) \subset\left(\tau_{1}, \tau_{2}\right)-b L \operatorname{cl}\left(f^{-1}(A)\right) \Longrightarrow f^{-1}\left(\sigma_{i}-\operatorname{cl}(A)\right) \subset\left(\tau_{1}, \tau_{2}\right)-b L \operatorname{cl}\left(f^{-1}(A)\right) .
$$

(b) $\Longrightarrow$ (a) Let $A$ be a subset of $Y$ and $B$ be a $\left(\tau_{1}, \tau_{2}\right)$ - $b$-locally closed set in $X$ such that $f^{-1}(A) \subset$ $B$. We have $A \subset \sigma_{i}-\operatorname{cl}(A)$ and $\sigma_{i}-\operatorname{cl}(A)$ is $\sigma_{i}$-closed.

Therefore by hypothesis, $f^{-1}\left(\sigma_{i}-\operatorname{cl}(A)\right) \subset\left(\tau_{1}, \tau_{2}\right)-b L \operatorname{cl}\left(f^{-1}(A)\right) \subset\left(\tau_{1}, \tau_{2}\right)-b L \operatorname{cl}(B)=B \Longrightarrow$ $f^{-1}\left(\sigma_{i}-\operatorname{cl}(A)\right) \subset B$.

Hence by Theorem 2.4, we have $f$ is pairwise $b$-locally open mapping.

\section{Pairwise $b$-locally closed mapping}

Definition 3.1. Let $\left(X, \tau_{1}, \tau_{2}\right)$ and $\left(Y, \sigma_{1}, \sigma_{2}\right)$ be two bitopological spaces. A function $\mathrm{f}:\left(X, \tau_{1}\right.$, $\left.\tau_{2}\right) \rightarrow\left(Y, \sigma_{1}, \sigma_{2}\right)$ is called pairwise $b$-locally closed (in short, pairwise $b L C$ ) mapping if the image of each $\left(\tau_{1}, \tau_{2}\right)$ - $b$-locally closed set in $X$ is $\sigma_{i}$-closed set in $Y$, where $i=1,2$.

Example 3.1. Let $X=Y=\{a, b, c\}, \tau_{1}=\{\varnothing,\{b\}, X\}, \tau_{2}=\{\varnothing,\{a\}, X\}, \sigma_{1}=\{\varnothing,\{a\},\{a, b\}, Y\}, \sigma_{2}=$ $\{\varnothing,\{a\},\{a, b\},\{a, c\}, Y\}$. Consider the function $f:\left(X, \tau_{1}, \tau_{2}\right) \rightarrow\left(Y, \sigma_{1}, \sigma_{2}\right)$ defined by $f(a)=b$, 
$f(b)=c, f(c)=c$. Here $\left(\tau_{1}, \tau_{2}\right)-b L C$ sets are $\{\varnothing,\{b\},\{c\},\{a, b\},\{b, c\}, X\}$ and image of each $\left(\tau_{1}, \tau_{2}\right)-b L C$ sets are $\sigma_{1}$-closed. Also $\left(\tau_{2}, \tau_{1}\right)$-bLC sets are $\{\varnothing,\{a\},\{c\},\{a, b\},\{a, c\}, X\}$ and image of each $\left(\tau_{2}, \tau_{1}\right)$-bLC sets are $\sigma_{2}$-closed. Hence $f$ is pairwise $b$-locally closed mapping.

Theorem 3.1. Let $f:\left(X, \tau_{1}, \tau_{2}\right) \rightarrow\left(Y, \sigma_{1}, \sigma_{2}\right)$ be a mapping between two bitopological spaces. Then the following are equivalent:

(a) $f$ is pairwise b-locally closed mapping.

(b) $\sigma_{i}-\operatorname{cl}(f(A)) \subset f\left(\left(\tau_{1}, \tau_{2}\right)-b L \operatorname{cl}(A)\right)$, for each subset $A$ of $X$, where $i=1,2$.

Proof. (a) $\Longrightarrow$ (b) Let $f$ be pairwise $b$-locally closed mapping. Then for any subset $A$ of $X$ we have $A \subset\left(\tau_{1}, \tau_{2}\right)-b L \operatorname{cl}(A)$ and $\left(\tau_{1}, \tau_{2}\right)-b L \operatorname{cl}(A)$ is $\left(\tau_{1}, \tau_{2}\right)$-b-locally closed set in $X$. Thus $f(A) \subset f\left(\left(\tau_{1}, \tau_{2}\right)-b L \mathrm{cl}(A)\right)$.

By assumption we obtain $f\left(\left(\tau_{1}, \tau_{2}\right)-b L \operatorname{cl}(A)\right)$ is $\sigma_{i}$-closed.

Hence $\sigma_{i}-\operatorname{cl}(f(A)) \subset f\left(\left(\tau_{1}, \tau_{2}\right)-b L \operatorname{cl}(A)\right)$.

(b) $\Longrightarrow$ (a) Let $A$ be a $\left(\tau_{1}, \tau_{2}\right)$-b-locally closed set in $X$. We have $f(A) \subset \sigma_{i}$-cl $(f(A))$. By hypothesis, $\sigma_{i}-\operatorname{cl}(f(A)) \subset f\left(\left(\tau_{1}, \tau_{2}\right)-b L \operatorname{cl}(A)\right) \Longrightarrow \sigma_{i}-\operatorname{cl}(f(A)) \subset f(A)$.

Hence $f(A)$ is $\sigma_{i}$-closed in $Y$ and therefore $f$ is pairwise $b$-locally closed mapping.

Theorem 3.2. Let $f:\left(X, \tau_{1}, \tau_{2}\right) \rightarrow\left(Y, \sigma_{1}, \sigma_{2}\right)$ be a mapping. Then the following are equivalent. (a) $f$ be pairwise b-locally closed mapping.

(b) For any subset $A$ of $Y$ and for any $\left(\tau_{1}, \tau_{2}\right)-b$-locally open set $B$ in $X$ such that $f^{-1}(A) \subset B$, then there exists a $\sigma_{i}$-open set $C$ containing $A$ such that $f^{-1}(C) \subset B$, where $i=1,2$.

Proof. The proof is straightforward, therefore omitted.

Definition 3.2 ([8]). A function $f:\left(X, \tau_{1}, \tau_{2}\right) \rightarrow\left(Y, \sigma_{1}, \sigma_{2}\right)$ is pairwise $b$-locally closed irresolute if $f^{-1}(A) \in\left(\tau_{1}, \tau_{2}\right)$-bLC $(X)$ for every $A \in\left(\sigma_{1}, \sigma_{2}\right)-b L C(Y)$.

Theorem 3.3. Let $f:\left(X, \tau_{1}, \tau_{2}\right) \rightarrow\left(Y, \sigma_{1}, \sigma_{2}\right)$ and $g:\left(Y, \sigma_{1}, \sigma_{2}\right) \rightarrow\left(Z, \theta_{1}, \theta_{2}\right)$ be two functions such that $g_{0} f: X \rightarrow Z$ is pairwise b-locally closed mapping. If $f$ is pairwise b-locally closed irresolute surjection, then $g$ is pairwise b-locally closed mapping.

Proof. Suppose that, $A$ be $\left(\sigma_{1}, \sigma_{2}\right)$-b-locally closed set in $Y$. Since $f$ is pairwise $b$-locally closed irresolute, therefore $f^{-1}(A)$ is $\left(\tau_{1}, \tau_{2}\right)$-b-locally closed set in $X$.

Since $g_{0} f$ is pairwise $b$-locally closed map and $f$ is surjective, we have $\left(g_{0} f\right)\left(f^{-1}(A)\right)$ is $\theta_{i}$-closed $\Longrightarrow g(A)$ is $\theta_{i}$-closed.

Hence $g$ is pairwise $b$-locally closed map. 


\section{Acknowledgement}

The authors thank the reviewer for the comments those improved the presentation of the paper.

\section{References}

[1] D. Andrijevic, On b-open sets, Mat Vesnik, 48 (1996), 59-64.

[2] M. Jelic, A decomposition of pairwise continuity, J. Inst. Math. Comput. Sci.Math. Ser., 3(1990), $25-29$.

[3] M. Jelic, On some mappings of bitopological spaces, Suppl. Rend. Circ., Math. Palermo (2), 29(1992), $483-494$.

[4] J. C. Kelly, Bitopological spaces, Proc, London Math. Soc., 13 (1963), 71-89.

[5] C. Kuratowski and Sierpinski, Surles difference edeux ensembles ferms, Tohoku Math. Jour., 20, (1921), 22-25.

[6] A. A. Nasef, b-locally closed sets and related topics, Chaous, Solitions \& Fractals, 12(2001), 1909-1915.

[7] W. J. Pervin, Connectedness in bitopological spaces, Indag. Math., 29(1967), 369-372.

[8] N. Rajesh, On b-locally closed sets in Bitopological spaces, Jour. Ind. Acad. Math., 30(2008), 551-556.

[9] B. C. Tripathy and D. J. Sarma, On b-locally open sets in Bitopological spaces, Kyungpook Math. Jour., $\mathbf{5 1}(2011), 429-433$.

[10] B. C. Tripathy and D. J. Sarma, On pairwise continuous functions in Bitopological spaces, Acta Sci. Technol., 35(2013), (in press).

Mathematical Sciences Division, Institute of Advanced Study in Science and Technology, Paschim Boragaon, Garchuk; Guwahati - 781035, Assam, India.

E-mail: tripathybc@yahoo.com; tripathybc@rediffmail.com

E-mail: djs_math@rediffmail.com 